\title{
Evaluating the properties of ionic liquid at variable temperatures and pressures by QSPR
}

\author{
Shuying Zhang ${ }^{1}$, Qingzhu Jia ${ }^{1}$, Fangyou Yan ${ }^{2}$, Xia Shuqian ${ }^{3}$, and Qiang Wang ${ }^{1}$ \\ ${ }^{1}$ Tianjin University of Science and Technology \\ ${ }^{2}$ Affiliation not available \\ ${ }^{3}$ Tianjin University
}

September 16, 2020

\begin{abstract}
ILs thermodynamic properties at variable temperature and pressure, such as density, viscosity and thermal conductivity, are necessarily basal parameters of chemical engineering process. In this paper, some norm descriptors-based QSPR models are established to predict the properties of ILs at variable temperature and pressure. The f-T-P models are developed with 9020 data points of 314 ILs for density, 7342 data points of 351 ILs for viscosity and 608 data points of 87 ILs for thermal conductivity. These models have satisfactory statistical results for the calculation of ILs properties. The validation analysis shows that these QSPR models have good stability and predictability. And the norm descriptors are universal for predicting the properties of ILs. Moreover, these QSPR models are applied to predict parameters of f-T-P models for 16329 ILs generated by combing the cations and anions in the dataset, which might be valuable and further used handily by other chemists.
\end{abstract}

\section{Introduction}

Ionic liquids (ILs) are a kind of common low melting point organic salts. Recently, ILs have attracted much attention for their unique physical and chemical properties, such as good thermal stability, conductivity, wide electrochemical window, incombustibility and recyclability. ${ }^{1,2}$ Because of these characteristics, ILs have been successfully applied in the fields of electrochemistry, ${ }^{3-5}$ separation and extraction procedures, ${ }^{6-8}$ biological field ${ }^{9,10}$ and chemical engineering. ${ }^{2,11}$

The thermodynamic properties of ILs basically determine and affect their applications. Density $(\rho)$ is indispensable in the process design of material science and chemical engineering. In particular, the density of ILs can be used to calculate other properties (viscosity, surface tension, thermal conductivity, etc.). The viscosity $(\eta)$ of ILs can be used to determine the feasible operation conditions of the actual chemical process, such as fluids pumping and stirring, liquid-liquid extraction, distillation process. ${ }^{12}$ Thermal conductivity $(\lambda$ ) is very important for obtaining the heat transfer coefficient of fluid, which is essential for the design of heat transfer fluid and equipment. ${ }^{13,14}$ Until now, large amount of ILs have been synthesized by a variety of cations, anions and substituents. ${ }^{15}$ However, the existing experimental properties data of ILs cannot meet the requirements for guiding chemical industrial applications. Moreover, density, viscosity and thermal conductivity are significantly affected by the temperature and the pressure, and it is even impossible for obtaining all these experiment data under different temperatures and pressures through the time-consuming and expensive experimental methods. Therefore, it is urgent to provide a model to calculate these characteristics of ILs under variable temperature and pressure as an alternative to the experimental measurement.

To date, different methods have been reported in literatures to calculate the properties of ILs. ${ }^{16-20}$ These methods are mainly based on: (i) the group contribution model (GCM), and (ii) the quantitative structureproperty relationship (QSPR). GCM is a very important method to predict various physical and thermo- 
dynamic properties with satisfy prediction results ${ }^{21}$. For the thermal conductivity of ionic liquids, Lazzús ${ }^{22}$ established a GCM with 400 data point of 41 ILs at a wide range of temperature (253-395 K) and pressure $(0.1-20 \mathrm{MPa})\left(R^{2}=0.9843, A A R D=2.12 \%\right)$. Recently, Chen et al. ${ }^{16}$ developed a GCM model to calculate properties at variable temperatures of ILs including the density (7360 data points, 143 ILs) and the viscosity (1090 data points, 76 ILs) with good prediction results. It is undeniable that these models provide accurate predictions for the physical properties of ILs. However, GCM depends on the group contribution value, and due to the lack of some group contribution values, the properties of some ILs cannot be calculated for some ILs.

In the last decade, QSPR has been profusely employed to study the properties of ILs, such as heat capacity, ${ }^{23-25}$ viscosity, ${ }^{26-29}$ thermal conductivity, ${ }^{30,31}$ surface tension, ${ }^{32,33}$ and toxicity. ${ }^{34-37}{ }^{3}$ Lazzús ${ }^{38}$ estimated the density in a wide of temperature $(253-473 \mathrm{~K})$ and pressure $(0.1-250 \mathrm{MPa})$ range with satisfactory results $(A A R D=2.00 \%)$. Yan et al. ${ }^{39}$ established a QSPR model to predict the density of ILs under variable temperature and pressure by using topological index with $A A R D$ of $0.42 \%$. Recently, AguirrePaduszynski ${ }^{40}$ established a reference term model and a modified QSPR model to predict the density (temperature (217$473 \mathrm{~K}$ ) and pressure (0.1-250 MPa)) of ILs, and good results could be obtained with AARD of $0.9 \%$. Paduszyński ${ }^{41}$ has proposed a new QSPR method for calculating ILs density and viscosity with temperature (217-473 K) and pressure (0.1-250 MPa) by MLR and LSSVM with good results.

Beckner et al. ${ }^{42}$ used neural networks to get a high-precision model to predict the ILs viscosity at variable temperatures and pressure with satisfactory results $(A A R D=7.10 \%)$. Zhao et al. ${ }^{43}$ established a new QSPR model using multiple linear regression (MLR) and support vector machine (SVM) algorithm to predict the viscosity of ILs at variable temperature $(253.15-395.32 \mathrm{~K})$ and pressure (0.1-300 MPa) with the overall AARD of $6.58 \%$. Yan et al. ${ }^{29}$ predicted the viscosity of ILs under a wide range of temperature $(253.15-573 \mathrm{~K})$ and pressure (0.06-300 MPa), and obtained satisfactory prediction results with AARD of $4.62 \%$.

In case of thermal conductivity of ILs, Chen et al. ${ }^{44}$ and Lazzus et al. ${ }^{45}$ proposed a QSPR model to predict the thermal conductivity of ILs under the condition of variable temperature (273.15-390 K) with AARD of $2.0 \%-2.3 \%$. He et al. ${ }^{46}$ presents a linear QSPR model based on the norm-indexes for predicting ILs thermal conductivity in a wide temperature $(273.15-355.07 \mathrm{~K})$ and pressure range $(0.1-20.0 \mathrm{MPa})$ with $A A R D$ of 1.45 $\%$.

Indeed, the above QSPR and GCM reference methods have achieved good results in predicting the properties of ILs. However, in their modelling process, the descriptors used to build the model were made up of anion and cation descriptors, and their interactions were often neglected to some extent. In order to study the interaction between cations and anions, our previous work regarded the interaction between cations and anions as a mathematical formula composed of ion descriptors. ${ }^{37,47}$ Compared with the results in the above literatures, the accuracy of our previous works on the interaction between anions and cations have been significantly improved. Recently, Yan et al. ${ }^{25,48}$ proposed a new method to express the interaction between anions and cations by calculating descriptors from ILs molecule, which have been successfully applied for QSPR modelling for predicting the heat capacity and the eco-toxicity of ILs. The improved prediction accuracy and stability of the model further suggests that the interaction between anion and anion has an important impact on the properties of ILs, which is essential in the construction of the prediction model.

At present, there are hundreds of common cations and about 100 anions, and the combination of these anions and anions thus can produce tens of thousands of ILs. Although many literatures have reported QSPR models to predict thermodynamic properties, the descriptors used in these models need to be calculated by complicate software, which will lead to the inconvenience of model application. Therefore, the convenient and successful application of QSPR models for predicting the properties of density, viscosity and thermal conductivity is of great value for the design, development and application of ILs.

The focus of this work is to establish QSPR models for predicting the density, viscosity and thermal conductivity properties of ILs under variable temperature and pressure. There are four main works in this paper: (1) the $f$-T-P model of ILs under variable temperature and pressure was established; (2) the cationic, anionic 
and ILs descriptors were calculated to build the QSPR model; (3) three QSPR models were established for three properties of ILs at variable temperatures and pressures; (4) $f$-T-P model parameters of 16329 ILs were predicted by the QSPR models.

\section{Methodology}

\subsection{Database}

A comprehensive experimental dataset was established by collecting the experimental data of three properties (density, viscosity, thermal conductivity) of ILs from the Ionic Liquids database provided by the National Institute of Standards and Technology (NIST) (https://ilthermo.boulder.nist.gov/index.html) and papers of Yan et al. and He et al. ${ }^{29,39,46} 9020$ density $(\rho)$ data points for 315 ILs, 7342 viscosity $(\rho)$ data points for 351 ILs and 608 thermal conductivity $(\lambda)$ data points for 87 ILs are covered in the dataset. For density, viscosity and thermal conductivity, the temperature and pressure ranges are $(253.15-473.15 \mathrm{~K})$ and $(0.1-250$ $\mathrm{MPa}),(253.15-438.15 \mathrm{~K})$ and $(0.06-300 \mathrm{MPa}),(273.15-390 \mathrm{~K})$ and (0.1-20.0 MPa), respectively.

The ILs are based on various cations such as imidazoulium ( $\mathrm{Im}$ ), pyridinium (Py), ammonium $(\mathrm{Am})$, pyrrolidinium (Pyr), phosphonium $(\mathrm{Ph})$, piperidinium (Pip), morpholinium (Mor), sulphonium (S), pyrrolidonium (N), diethylthiouronium (Thiur), 1,8-diazabicycloundecenium (Dbu), Noctylisoquinolinium (Quin), Caprolactam $(\mathrm{Cp})$. And anions included tetrafluoroborate $\left[\left(\mathrm{BF}_{4}\right)^{-}\right]$, hexafluorophosphate $\left[\left(\mathrm{PF}_{6}\right)^{-}\right]$, dicyanamide $\left[\left(\mathrm{N}(\mathrm{CN})_{2}\right)^{-}\right]$, tetracyanoborate $\left[\left(\mathrm{B}(\mathrm{CN})_{4}\right)^{-}\right]$, trifluoroacetate $\left[\left(\mathrm{C}(\mathrm{CN})_{3}\right)^{-}\right]$, alkyl-phosphate $\left[\left(\mathrm{RPO}_{4}\right)^{-}\right]$, bis $\left[(\right.$perfluoroethyl $)$sulfonyl]imide $\left[\left(\mathrm{Pf}_{2} \mathrm{~N}\right)^{-}\right]$, alkoxy-alkylsulfates $\left[\left(\mathrm{RSO}_{3}\right)^{-}\right]$, tris(pentafluoroethyl)trifluorophosphate $\left[(\mathrm{FAP})^{-}\right]$, alkyl-sulfate $\left[\left(\mathrm{RSO}_{4}\right)^{-}\right]$, thiocyanate $\left[(\mathrm{SCN})^{-}\right]$, carboxylicacid $\left[\left(\mathrm{RCO}_{2}\right)^{-}\right]$, bis $\left[(\right.$trifluoromethyl $)$sulfonyl] imide $\left[\left(\mathrm{Tf}_{2} \mathrm{~N}\right)^{-}\right]$, halogen $\left[(\mathrm{X})^{-}\right], 1,2,3$-triazolide $\left[(\text { Triz })^{-}\right]$, Tetrazolide $\left[(\text { Tetra })^{-}\right]$, difluoromono[1,2-oxalato $\left.(2-)-\mathrm{O}, \mathrm{O}^{\prime}\right]$ borate $\left[\left(\mathrm{RBF}_{2}\right)^{-}\right], 4$-nitropyrazolide $\left[\left(4-\mathrm{NO}_{2} \text { pyra }\right)^{-}\right]$, phenolate $\left[(\mathrm{Phe})^{-}\right], 2$-(cyano)pyrrolide $\left[(2-\mathrm{CNpyr})^{-}\right]$. The ILs name together with corresponding experimental values of density, viscosity and thermal conductivity were shown in Supporting Information Tables S1 ${ }^{\sim} 3$.

\section{$2.2 f-T-P$ model}

In this work, the $f-T-P$ models are established to describe the relationship of properties (density, viscosity and thermal conductivity) with temperature and pressure. The $f-T-P(\rho-T-\Pi, \eta-T-\Pi, \lambda-T-\Pi)$ model shown as Eq. (1) was used at present for this study. The fitting parameters $\alpha, \beta, \gamma$ and $\chi$, used to calculate the density, viscosity and thermal conductivity of ILs are given in Supporting Information Figures S1 S3.

In structure-property relationship, $a$ is related to the structures of ILs and the parameters $\beta, \gamma$ and $\chi$ are obtained by two methods: (1) $\beta, \gamma$ and $\chi$ are set as constants for all ILs; (2) $\beta, \gamma$ and $\chi$ are variables related to the molecular structure. To compare these two approaches, the density, viscosity and thermal conductivity samples with multiple data points are fitted using Eq. (1) by the above two methods. The calculated results are depicted in Figures 1-3. It can be seen that the second method results in Figure 1 (b), Figure 2 (b) and Figure 3 (b) are better than the first method results in Figure 1 (a), Figure 2 (a) and Figure 3 (a). The correlations between the calculated and experimental values for each sample using the second method are presented in the supporting information. Compared with the first method, the average absolute relative deviation (AARD) of the second method reduced by $76 \%, 79 \%$ and $70 \%$ for $\rho, \eta$ and $\lambda$, respectively. Accordingly, the above calculation results demonstrated that $\beta, \gamma$ and $\chi$ adjusted by the molecular structures are necessary and efficient. 

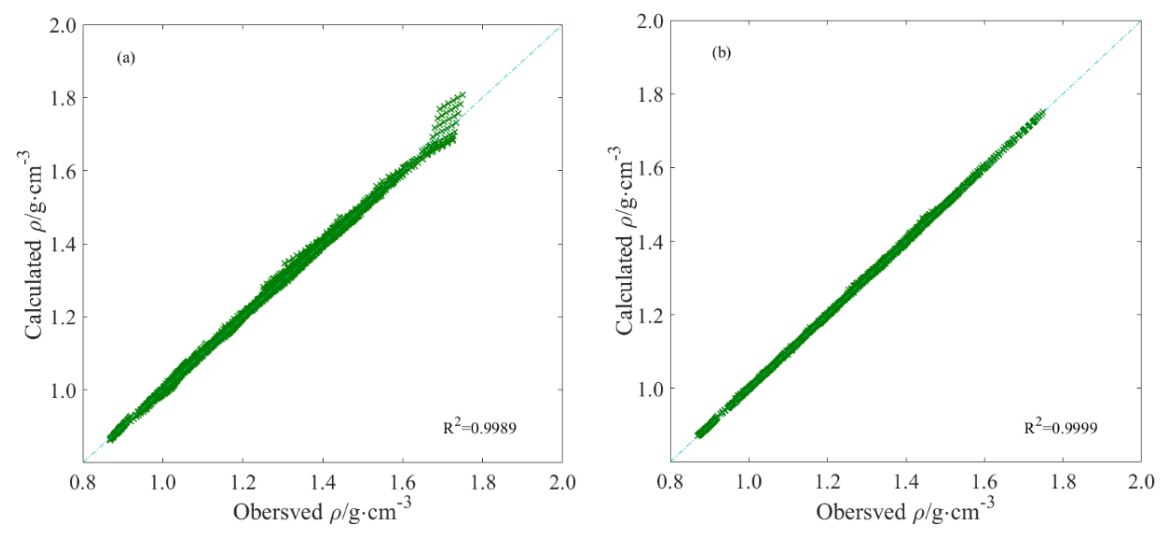

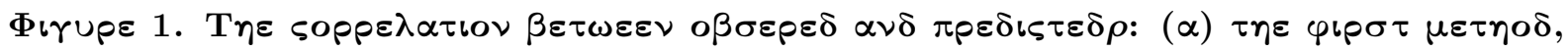
(b) the second method.
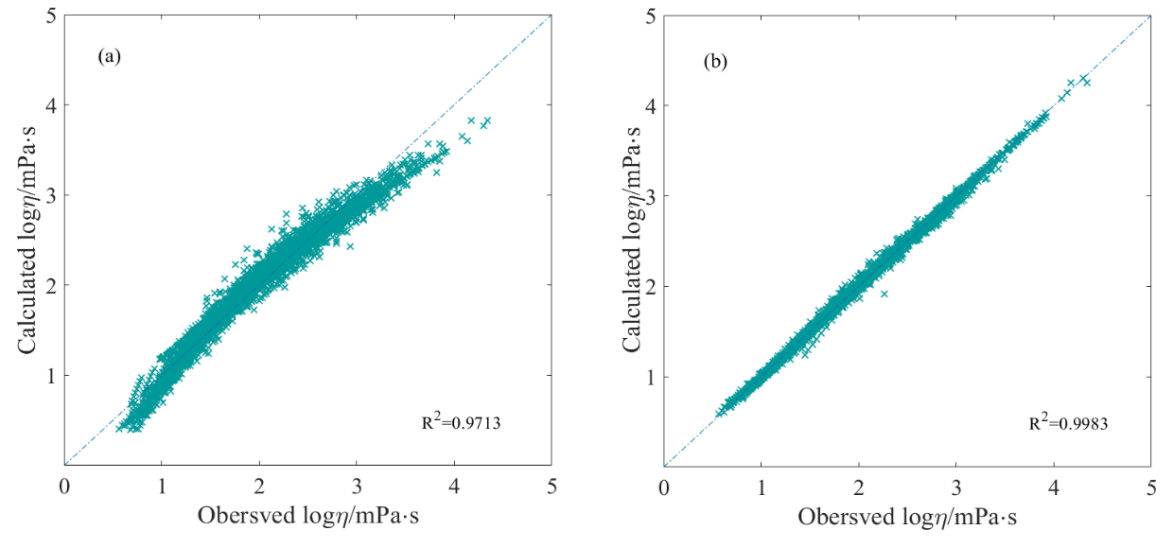

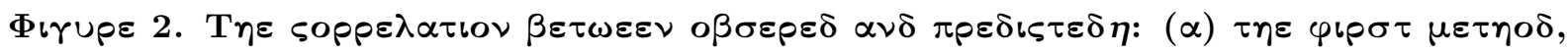
(b) the second method.
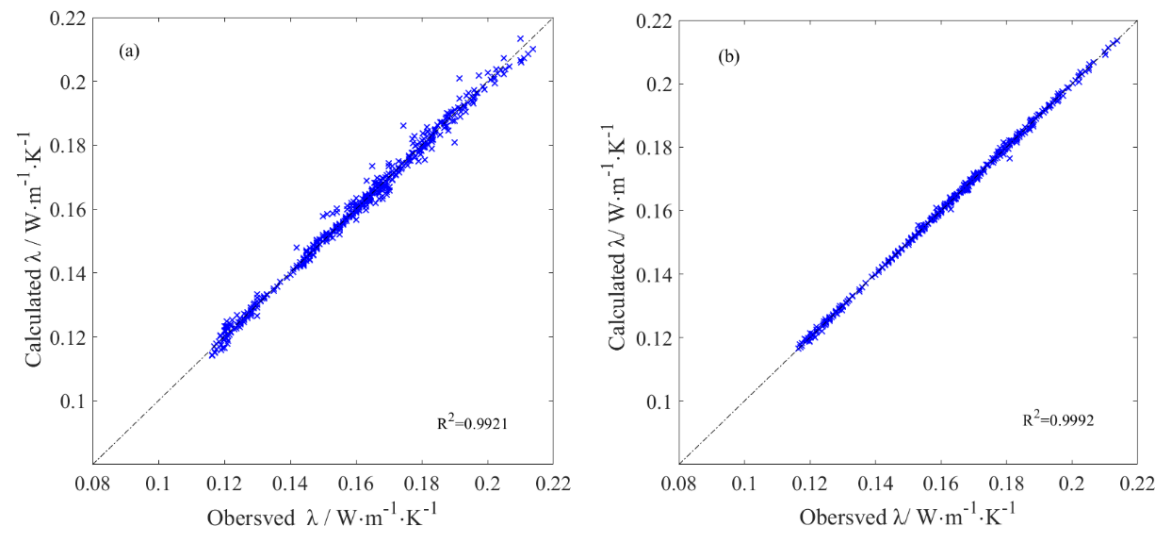

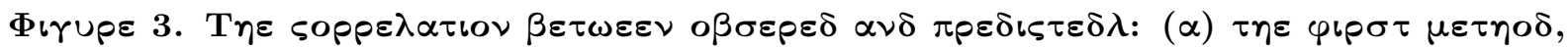
(b) the second method. 


\subsection{Norm descriptors proposed}

The structure of ILs is the key factor to determine the properties of ionic liquid. With the HyperChem 7.0 software (http://www.hyper.com/), the $a b$ initio method at the ST0-3G level was used to optimize the structure of cation, anion and IL. In order to clearly reflect the position relationship of atoms, the step matrix $(M A, M B, M C M S)$ and Euclidean space distance matrix $(M D)$ are expressed as follows:

In order to reveal the characters of atoms in molecules, the property matrices based on a series of atomic properties are as Eqs. (7-13):

Where, $a w, m w, e, i p, r$, ne, el and $q$ represent atom weight, molecular weight, electronegativity, ionization potential, atomic radius, outermost number of electrons, number of electron layers and atom charge, respectively.

The atomic distribution matrix $(M)$, used for density, viscosity and thermal conductivity, are shown in Supporting Information Tables C1-C3. The $M$ is composed of the distance matrix or the combination of step matrix and property matrix, which reflects the relationship between atoms and the special contribution of each atom. Then, the norm descriptors $(I)$ were calculated from the $M$ through the following formulas (Eqs. (14-17)).

\section{Hosted file}

image8.wmf available at https://authorea.com/users/359155/articles/481358-evaluating-theproperties-of-ionic-liquid-at-variable-temperatures-and-pressures-by-qspr

Where, $\lambda_{\mathrm{i}}$ is the matrix eigenvalue, and the is the Hermite matrix.

\subsection{Model Validation}

These QSPR models were quantitatively assessed by several statistical criteria as relative deviation $(R D)$, the Fisher significance parameter $(F)$ and the average absolute relative deviation percentage $(A A R D \%)$. The predictability of the model is supported by $R_{\text {training }}^{2}$ for the training set and $R_{\text {testing }}^{2}$ for the testing set. In addition, Leave-one-out (LOO) validation was utilized to evaluate the robustness of this QSPR model $\left(Q^{2}\right)$. $Y$-randomization test was utilized to avoid the possibility of chance correlation in the modelling work. ${ }^{32,49}$

\section{Hosted file}

image9.wmf available at https://authorea.com/users/359155/articles/481358-evaluating-theproperties-of-ionic-liquid-at-variable-temperatures-and-pressures-by-qspr

\section{Hosted file}

image10.wmf available at https://authorea.com/users/359155/articles/481358-evaluating-theproperties-of-ionic-liquid-at-variable-temperatures-and-pressures-by-qspr

\section{Hosted file}

image11.wmf available at https://authorea.com/users/359155/articles/481358-evaluating-theproperties-of-ionic-liquid-at-variable-temperatures-and-pressures-by-qspr

Herein,

and

are the experimental and calculated values of ILs, respectively. $n$ is the total number of data points.

\subsection{Model Application}

The stable QSPR model could be used to predict the physical and chemical values of ILs. Based on the ILs studied in this work, 18630 ILs were obtained by combining 207 anions and 90 cations (Supporting 
Information Table S4). 16329 ILs with optimization structures are used to predict the parameters $(a, \beta, \gamma$ and $\chi$ ) of the $f-T-P$ model by using the three QSPR models for density, viscosity and thermal conductivity. Based on the experimental values range of density, viscosity and thermal conductivity, we assume that the reasonable range of predicted values is $50 \%$ of the minimum experimental value and $150 \%$ of the maximum experimental value. The coefficients $(a, \beta, \gamma$ and $\chi)$ of density, viscosity and thermal conductivity were predicted and provided in Supporting Information Tables S5-S7.

\section{Results and discussion}

\section{$3.1 \Delta \varepsilon \nu \sigma \iota \tau(\rho)$}

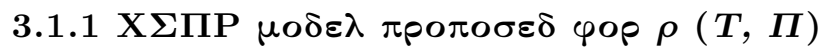

A new $\rho(T, P)$ model is established to estimate the temperature and pressure dependent $\rho$ of ILs, as shown in Eq. (20), and its parameter values are shown in Supporting Information Table C1.

$n=9020, R^{2}=0.9966, F=44685, Q^{2}=0.9965, A A R D=0.48 \%, R M S E=0.0097$

Where, $I_{\mathrm{ILs}}, I_{\mathrm{Ca}}$ and $I_{\text {An }}$ represent the norm descriptors of ILs, cation and anion, respectively.

The experimental and calculated $\rho$ values by the model (Eq. (20)) were shown in Supporting Information Table S1. The comparison between the observed value and the calculated value, as well as the residual distribution of the model and Leave-one-out cross-validation (LOO-CV) are shown in Figure 4. Figure 4 (a) indicated that that the model is accurate for most points distributed nearby, with $R^{2}$ of $0.9966, A A R D$ of $0.48 \%$ and $R M S E$ of 0.0097 . Figure 4 (b) suggested that about $96 \% \rho$ data points residuals range from -0.005 to 0.005 . Thus, it suggests that the model based on the norm index to calculate the density is stable and reliable.
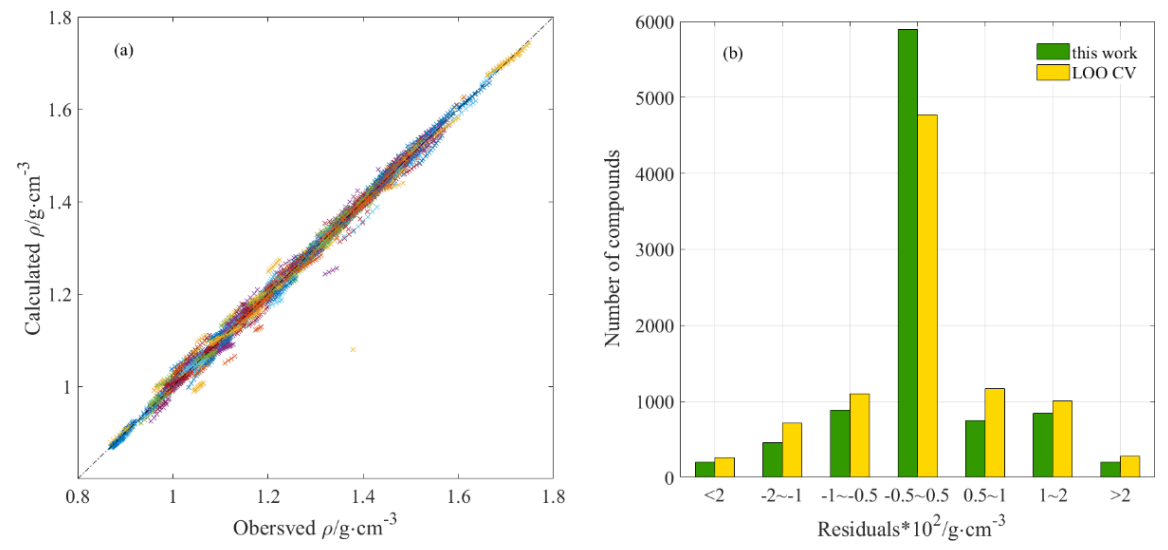

Figure 4. Observedvs. calculated values (a) and distribution of the residual (b) of density

314 ionic liquids can be divided into 13 types cations and 25 types anions. Figure 5 analyzes $A A R D$ and the $\rho$ data points of each type of ILs. It can be seen that the predicted $A A R D$ values of $[\mathrm{Am}]\left[\mathrm{RSO}_{4}\right]$ and $[\mathrm{Dbu}]\left[\mathrm{RSO}_{3}\right]$ are relatively large, which may lead to inaccurate prediction results for this two types ILs. However, most of the data point errors are within the acceptable range. Therefore, this model can be used to predict the density of ILs with different structures under different temperatures and pressures. 


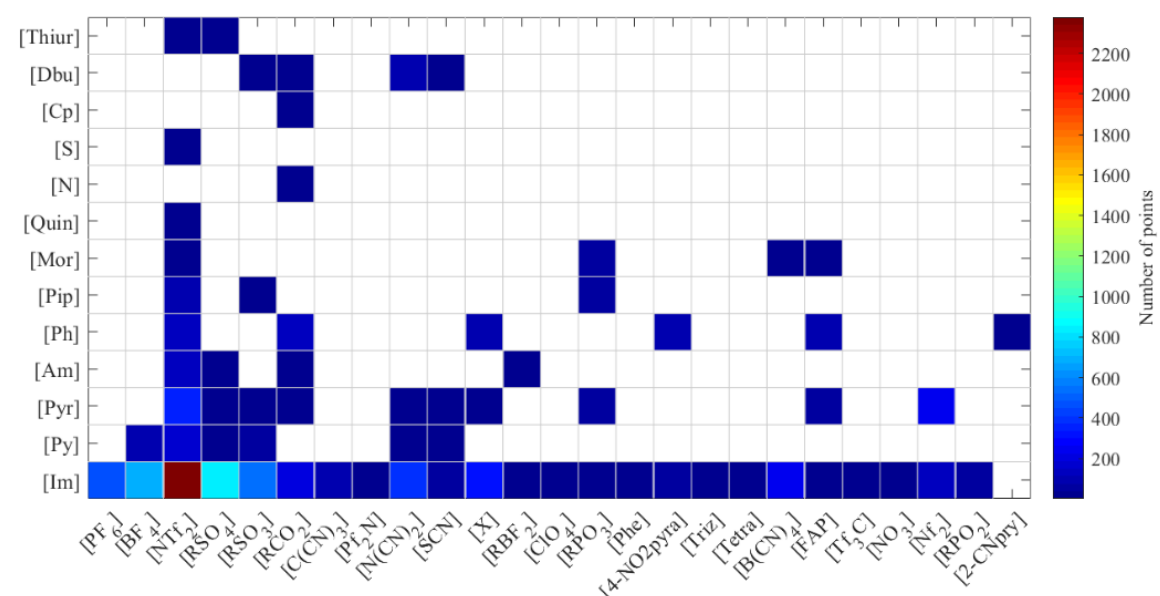

(a)

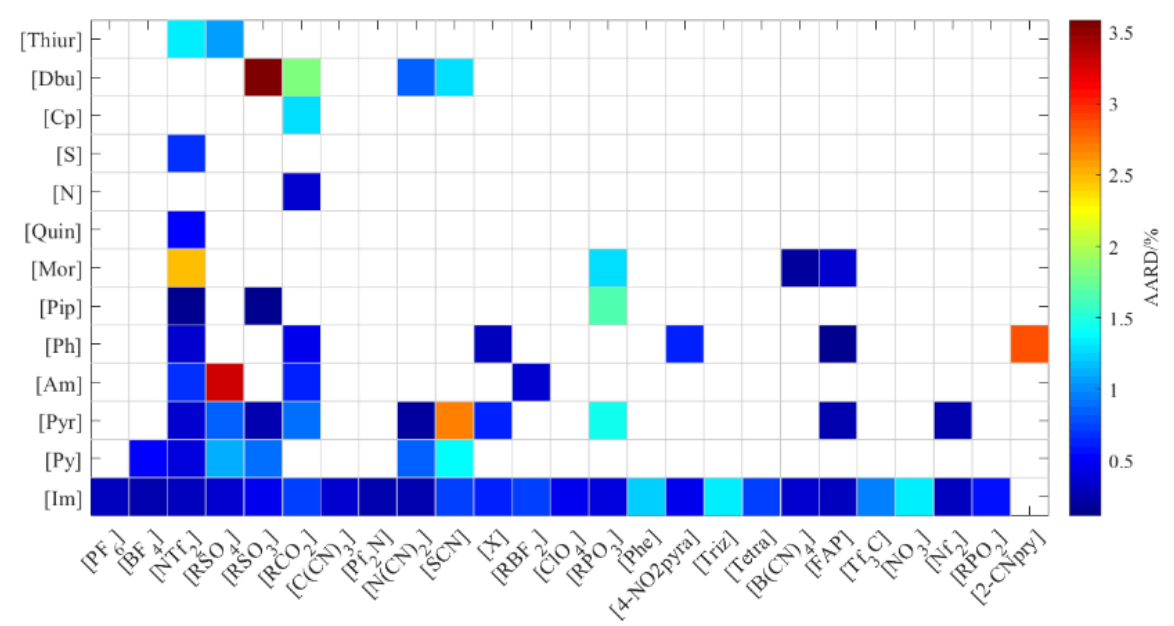

(b)

Figure 5. The numbers (a) and $A A R D \%$ (b) for each kind of ILs for density.

\subsubsection{Internal-validation and External-validation}

The robustness and predictability of the model were evaluated using the internal and external procedure. ${ }^{50}$ The validation parameters such as $R^{2}, Q^{2}$, AARD and $R M S E$ are listed in Table 1. As shown in Figure 6 (a), the good results of LOO-CV with high $Q^{2}$ of 0.9965 suggested the model's stability. For the external validation, the data set was randomly divided into the training set (7216 data points) and the testing set (1804 data points). The calculated and experimental $\rho$ values of the training set and the testing set were plotted in Figure 6 (b). The $R^{2}$ of the training set and testing set were 0.9954 and 0.9964 , respectively. The $A A R D$ value were $0.56 \%$ and $0.54 \%$, respectively. Results showed that the $\rho(T, P)$ model was stable and has good predictability. 

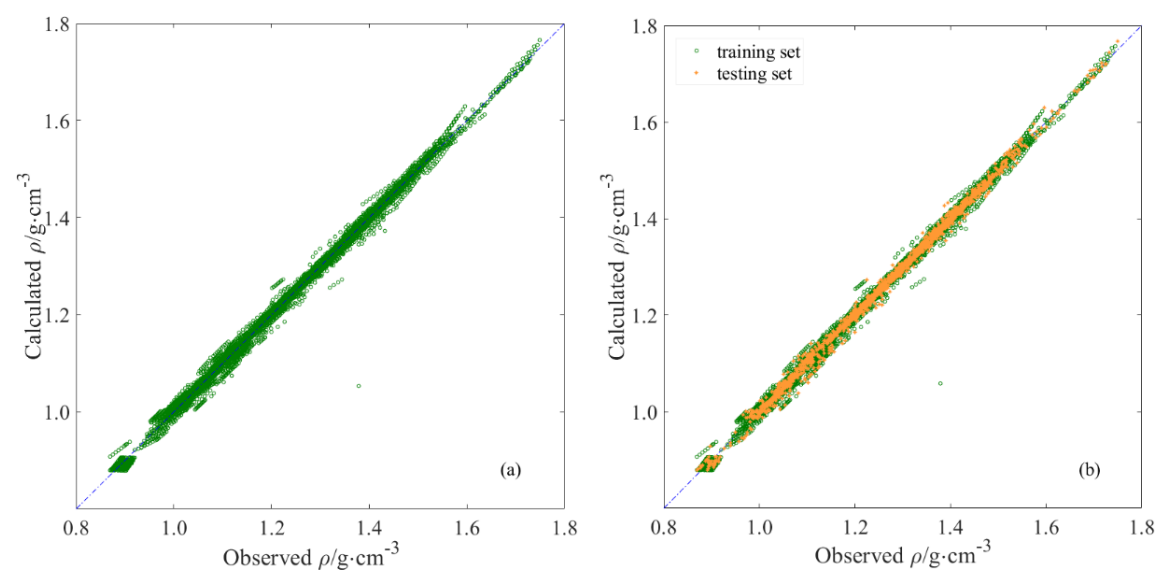

Figure 6. Scatter plots of the internal-validation (a) and external-validation (b).

Table 1. The results of internal-validation and external-validation for density

\begin{tabular}{lllll}
\hline Status & Data points & $R^{2}\left(Q^{2}\right)$ & $A A R D \%$ & $R M S E$ \\
\hline Model & 9020 & 0.9966 & 0.4764 & 0.0097 \\
LOO-CV & 9020 & 0.9965 & 0.4815 & 0.0110 \\
Training set & 7216 & 0.9954 & 0.5654 & 0.0101 \\
Testing set & 1804 & 0.9964 & 0.5464 & 0.0111 \\
\hline
\end{tabular}

\section{3. $1.3 \mathrm{Y}$-randomization test}

$Y$-randomized trials were conducted and Figure 7 shows the results of 1000 random validations. The $R_{\mathrm{r}}{ }^{2}$ and $Q \mathrm{r}^{2}$ of $Y$-type randomized test results were lower than 0.011 . Therefore, it can be considered that the $\rho(T, P)$ model is robust and is not developed by the chance correlation. 


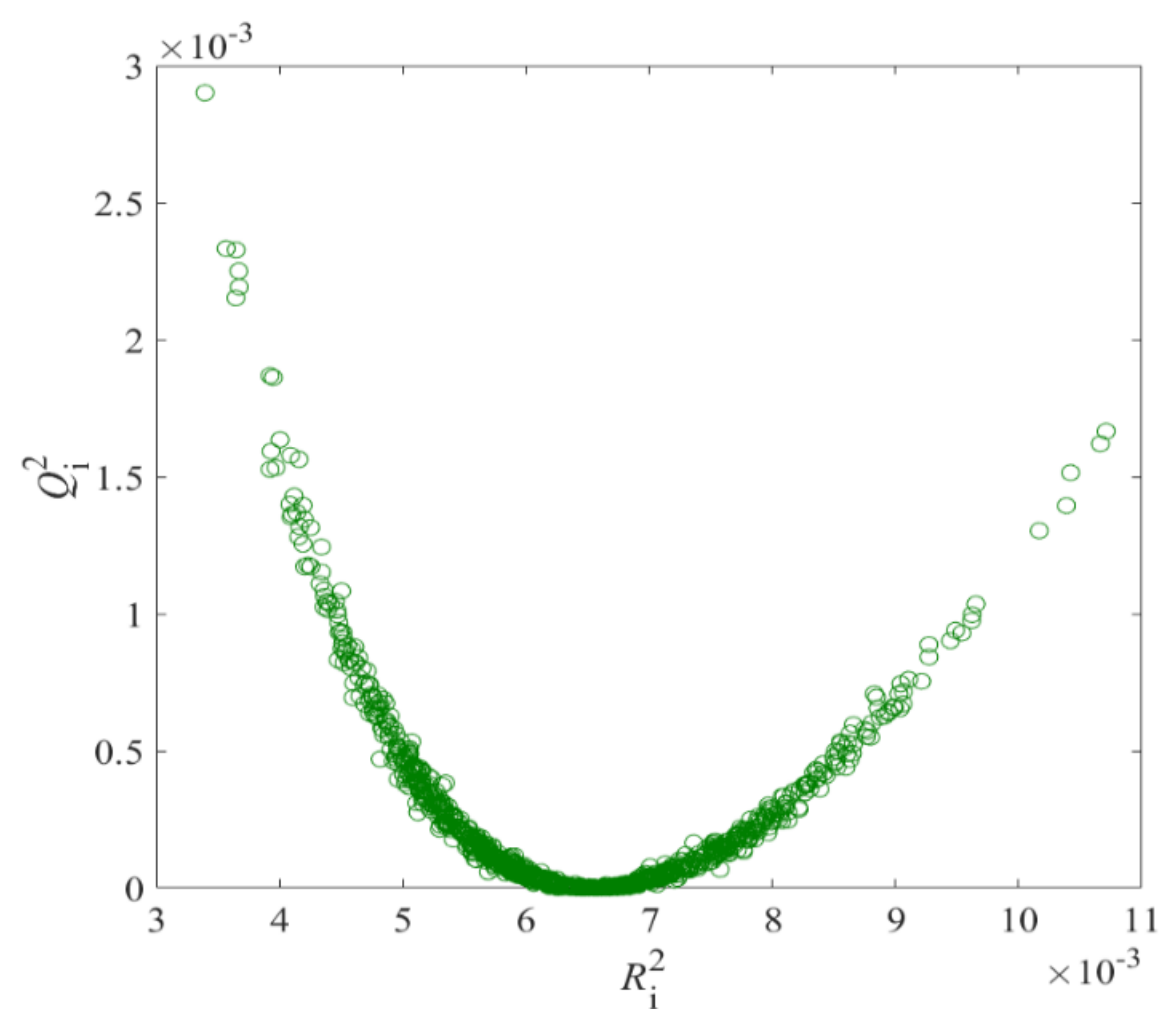

Figure 7. Results of the 1000 times $Y$-randomization test for density.

\subsubsection{Comparison with References}

Table 2. The comparisons of this work with references for the density

\begin{tabular}{llllllll}
\hline References & Method & D.P & ILs & T/K & P/MPa & $R^{2}$ & AARD (\%) \\
\hline Barati-Harooni et al. 2016 & GCM & 602 & 146 & $278-358$ & 0.1 & 0.9808 & 0.89 \\
Lazzús 201052 & GCM & 3530 & 76 & $258-393$ & $0.1-207$ & 0.9989 & 0.73 \\
Chen et al. 2019 $^{16}$ & GCM & 7360 & 143 & $273.15-473.15$ & $0.1-250$ & & 0.49 \\
Lazzús 2009 & QSPR & 3020 & 163 & $258-473$ & $0.1-207$ & 0.9307 & 2.00 \\
Yan et al. 201554 & QSPR & 5948 & 188 & $253.15-473.15$ & $0.1-250$ & 0.9980 & 0.42 \\
This work & QSPR & 9020 & 314 & $253.15-473.15$ & $0.1-250$ & 0.9966 & 0.48 \\
\hline
\end{tabular}

D.P is data points. $\mathrm{T}$ and $\mathrm{P}$ were temperature and pressure, respectively.

This model was compared with some other corresponding methods from literatures and the results were listed in Table 2. Based on GCM, Barati-Harooni et al. ${ }^{51}$ constructed a model to predict the density of ILs at variable temperature with $A A R D$ of $0.98 \%$. Lazzús ${ }^{52}$ and Chen et al. ${ }^{16}$ have made a good prediction for the density of ILs, in which the $A A R D$ value is about $0.73 \%$ and $0.49 \%$. For the QSPR model of Lazzús ${ }^{53}$, a moderate prediction result is obtained $(A A R D=2 \%)$. Obviously, the statistical results of these models are good. Although the $A A R D(0.48 \%)$ and $R^{2}(0.9966)$ of this work are slightly worse than those of prior studies ${ }^{54}$ the amount of ILs datasets in this paper is the larger. At the same time, a large number of ILs datasets with different structures are used for modeling in our work, and the satisfactory prediction results further confirm the robustness and reliability of the $\rho(T, P)$ model.

\subsubsection{The application of QSPR model}


The parameters of ILs $\rho$ model are located in Supporting Information Table S5. Figure 8 shows prediction results of the density of 16329 ILs at variable temperature $(273-573 \mathrm{~K})$ and pressure (0.1-10 MPa). Most of the predicted values for density are acceptable and reasonable, except those of [bmim][FS], [Hprthiur][I], $\left[\mathrm{mC}_{10} \mathrm{im}\right][\mathrm{Pf} 2 \mathrm{~N}],[\mathrm{N} 113(\mathrm{eOH})][\mathrm{FAP}]$ and $[\mathrm{odbu}]\left[\mathrm{C}_{8} \mathrm{SO}_{3}\right]$.

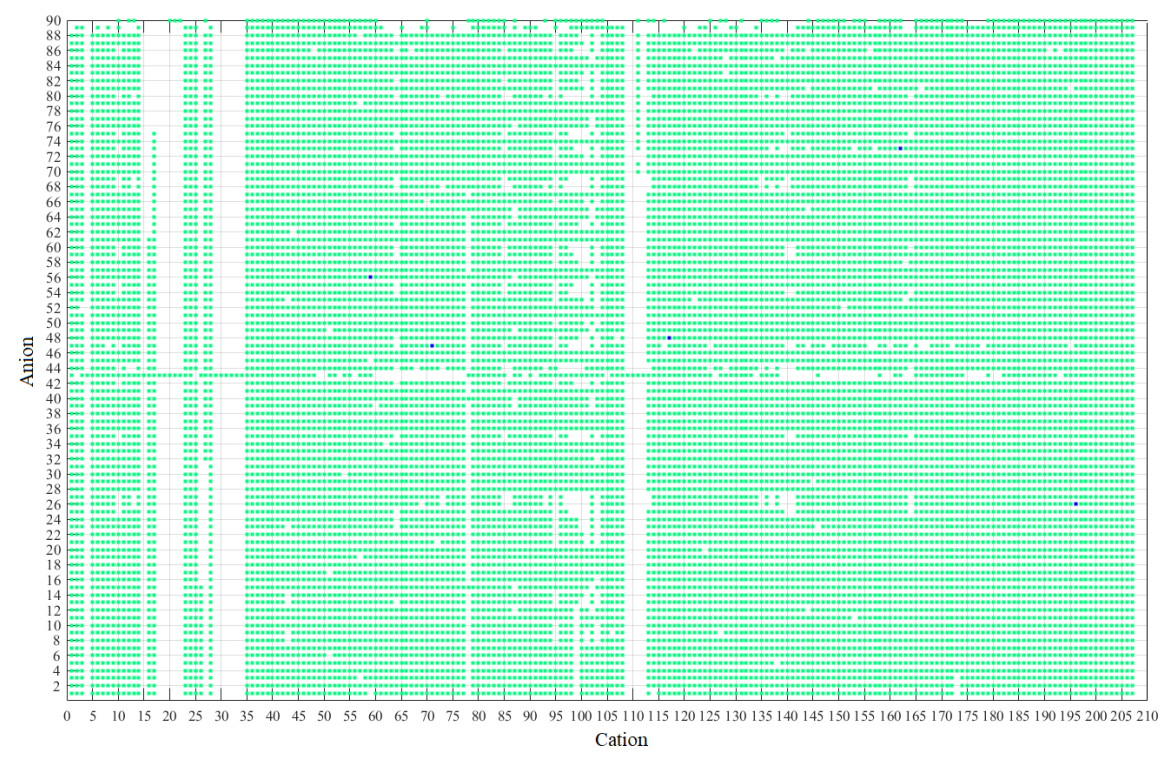

Figure 8. Prediction results of density: green-reasonable, blue-unreasonable.

\section{2. i $\sigma \varsigma 0 \sigma \iota \psi(\eta)$}

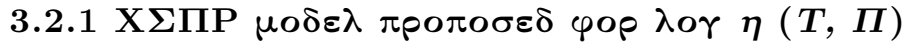

ILs $\eta$ model is expressed as Eq. (21). The coefficients of the model are shown in Supporting Information Table C2. The predicted and experimental $\log \eta$ values of ILs are listed in Supporting Information Table S2.

$n=7342, R^{2}=0.9676, F=4540, Q^{2}=0.9668, A A R D=3.91 \%, R M S E=0.1069$

Where, $I_{\mathrm{ILs}}, I_{\mathrm{Ca}}$ and $I_{\text {An }}$ represent the norm descriptors of ILs, cation and anion, respectively.

Additionally, the comparison between the observed and calculated $\log \eta$ values is shown in Figure 9 (a). The distribution of the residuals is shown in Figure 9 (b).
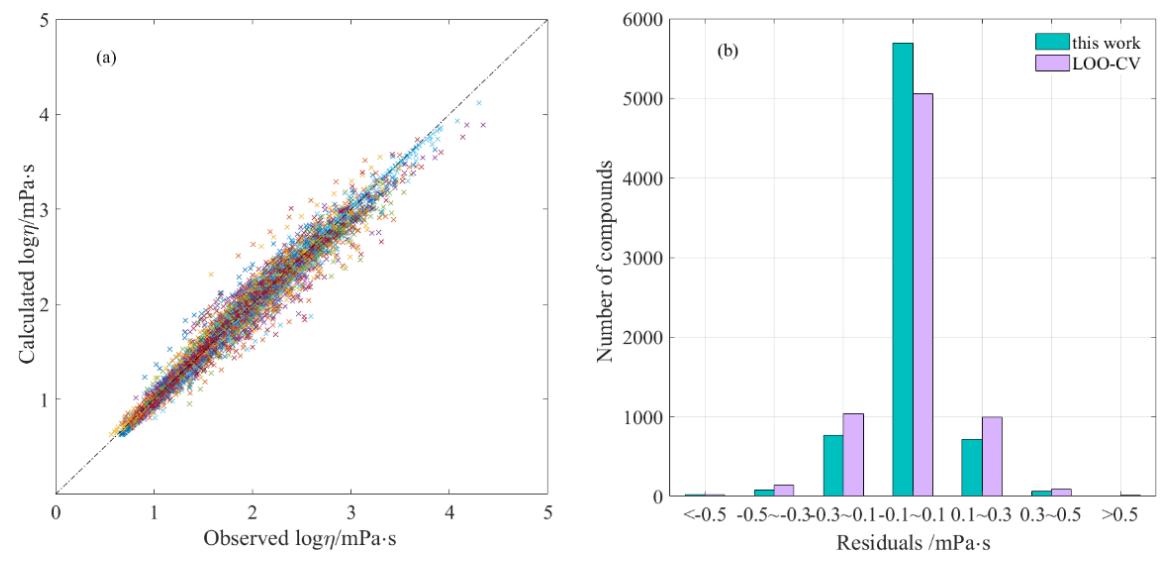


\section{Figure 9. Observed vs. calculated values (a) and distribution of the residual (b) of viscosity}

It can be seen that most of points are distributed near the diagonal, which means that the calculated value is close to the experimental value. The good prediction results are also denoted with $R^{2}$ of 0.9676 and the $A A R D$ of $3.91 \%$. Figure 9 (b) showed that the residual distribution of most ILs (about $80 \%$ ) $\log \eta$ data in the model are between $-0.5^{\sim} 0.5$, which further proves the reliability and stability of the model.

351 ionic liquids were divided into 11 categories of cations and 27 kinds of anions. A summary of $\eta$ estimation of ILs using QSPR method is given in Figure 10, in which the number of fitted data points and the AARD $\%$ of each type based ILs are provided.
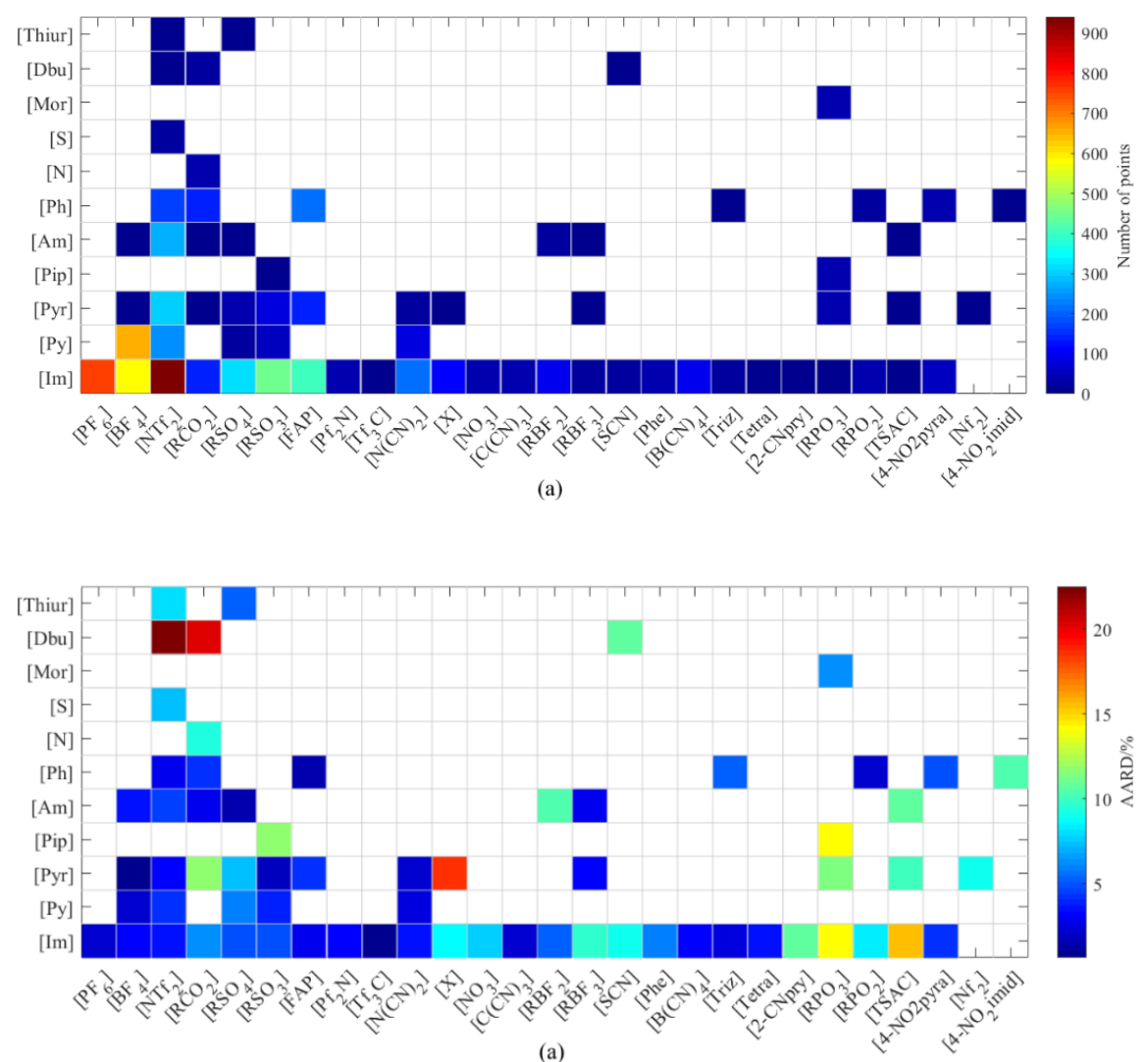

Figure 10. The numbers (a) and $A A R D \%$ (b) for each kind of ILs for viscosity

As shown in Figure 10 (b), all the $A A R D$ locates in acceptable range except for [Dbu] $\left[\mathrm{NTf}_{2}\right]$ and $[\mathrm{Dbu}]\left[\mathrm{RCO}_{2}\right]$, but this two types ILs only account for a small proportion in the whole data set. Accordingly, it could be concluded that the model is appropriate for predicting $\eta$ at variable temperature and pressure of ILs with various structures.

\subsubsection{Internal-validation and External-validation}

The Cross-validation calculated values versus experimental values of $\log \eta$ data were showed in Figure 11 (a). The statistical parameters for validation are listed in Table 3. The Figure 11 (a) shows that the experimental value is close to the $\log \eta$ value calculated by Cross-validation. Table 3 shows that the $Q^{2}(0.9668), A A R D$ $(3.95 \%)$ and RMSE (0.1276) are acceptable, which suggests the good stability and robustness of this $\eta$ model.

7432 data points were randomly divided into the training set (5874 data points) and the testing set (1468 data points). The calculated values for the training set and the testing set with the experimental values were 
showed in Figure 11 (b). The performance parameters for external validation results are listed in Table 3. External validation results showed that the $R^{2}$ of training set and testing set were 0.9535 and 0.9607 , and the $A A R D$ were $5.051 \%$ and $4.743 \%$, respectively. These result showed that the QSPR model based on norm descriptor was stable and has good predictive ability for $\eta$ prediction of ILs with diverse structures.
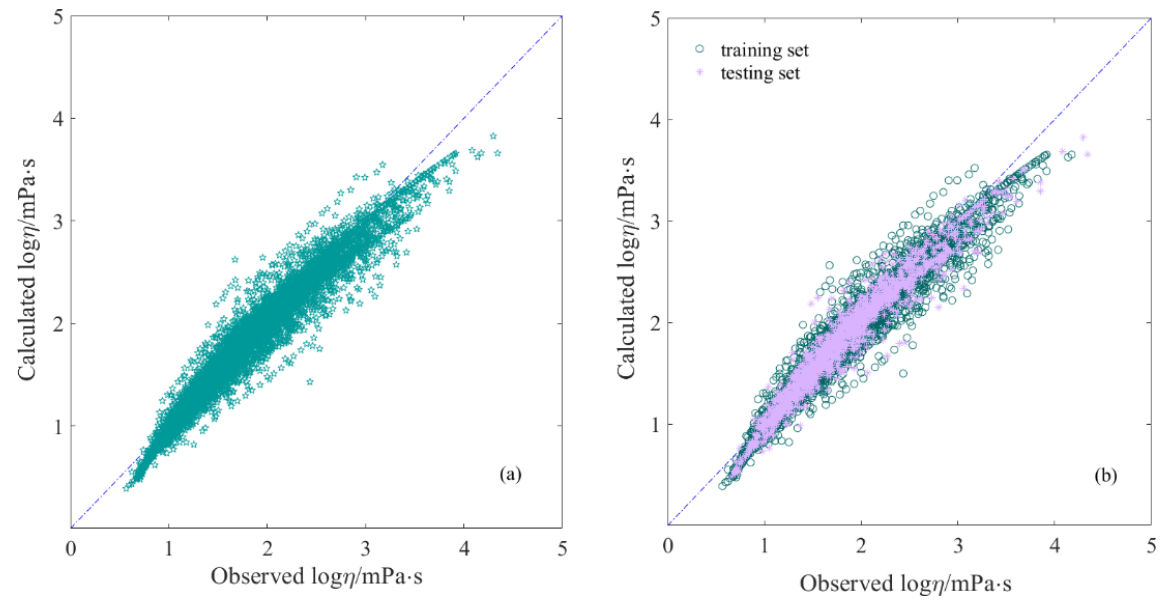

Figure 11. Scatter plots of the internal-validation (a) and external-validation (b)

Table 3. The results of internal-validation and external-validation for viscosity

\begin{tabular}{lllll}
\hline Status & Data points & $R^{2}\left(Q^{2}\right)$ & $A A R D \%$ & $R M S E$ \\
\hline Model & 7342 & 0.9676 & 3.914 & 0.1069 \\
LOO-CV & 7342 & 0.9668 & 3.951 & 0.1276 \\
Training set & 5874 & 0.9535 & 5.051 & 0.1285 \\
Testing set & 1468 & 0.9607 & 4.743 & 0.1166 \\
\hline
\end{tabular}

\subsubsection{Y-randomization test}

$1000 Y$-random verifications were repeated in this study and the results were showed in Figure 12. Figure 12 denoted that all $R_{\mathrm{i}}^{2}$ and $Q_{\mathrm{i}}^{2}$ values were lower than 0.012 , which indicated that this model was not established by accident. 


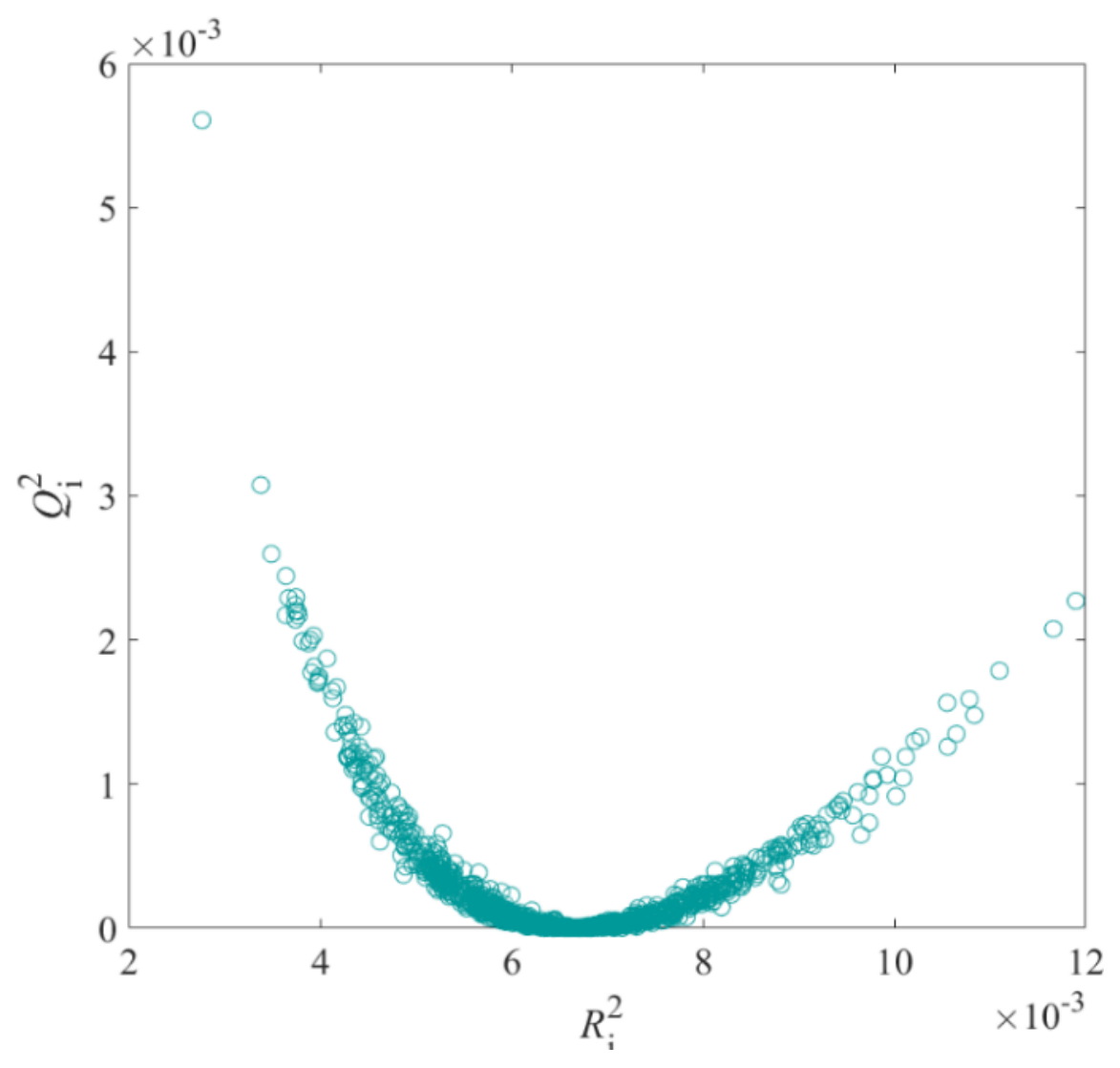

Figure 12. Results of the 1000 times Y-randomization test for viscosity.

\subsubsection{Comparison with References}

Table 4. The comparisons of this work with references for the viscosity

\begin{tabular}{llllllll}
\hline References & Model & D.P & ILs & T/K & P/MPa & $R^{2}$ & AARD (\%) \\
\hline Chen et al. 2019 & GCM & 1090 & 76 & $278.0-408.15$ & & & 3.58 \\
Beckner et al. 2018 & FF-ANN & 723 & 33 & $273.15-373.15$ & $0.06-0.16$ & & 7.10 \\
Zhao et al. 2015 & QSPR & 1502 & 89 & $253.15-395.32$ & $0.1-300$ & 0.9440 & 6.58 \\
Yan et al. 2018 & QSPR & 3228 & 349 & $253.15-573$ & $0.06-300$ & 0.9640 & 4.62 \\
This work & QSPR & 7342 & 351 & $253.15-438.15$ & $0.06-300$ & 0.9676 & 3.91 \\
\hline
\end{tabular}

Table 4 listed the comparison results between literatures and this work. Compared with the results of Chen et al. ${ }^{16}$ and Beckner et al. ${ }^{42}$, our model obtained good statistical results under a wider range of temperature and pressure. Compared with Zhao et al. ${ }^{55}$ and Yan et al. ${ }^{29}$, it was clear that our model has a larger amount of data and a wider variety of ILs with higher $R^{2}$ and lower $A A R D$, which further demonstrated that our model for predicting $\eta$ values was more precise.

\subsubsection{The application of QSPR model}

The parameters of viscosity model are located in Supporting Information Table S6. As shown in Figure 13, 16329 ILs are utilized for predicting the viscosity under variable temperature $(273-573 \mathrm{~K})$ and pressure (0.1-10 MPa) and the results are shown in Figure 13. As shown in Figure 13, most of the viscosity predictions 
of ILs are reasonable, except the viscosity prediction of [N113(eOH)][FAP], [bmim][FS], $\left[\mathrm{mC}_{10} \mathrm{im}\right][\mathrm{Pf} 2 \mathrm{~N}]$ and $[\mathrm{odbu}]\left[\mathrm{C}_{8} \mathrm{SO}_{3}\right]$ with the four blue spots.

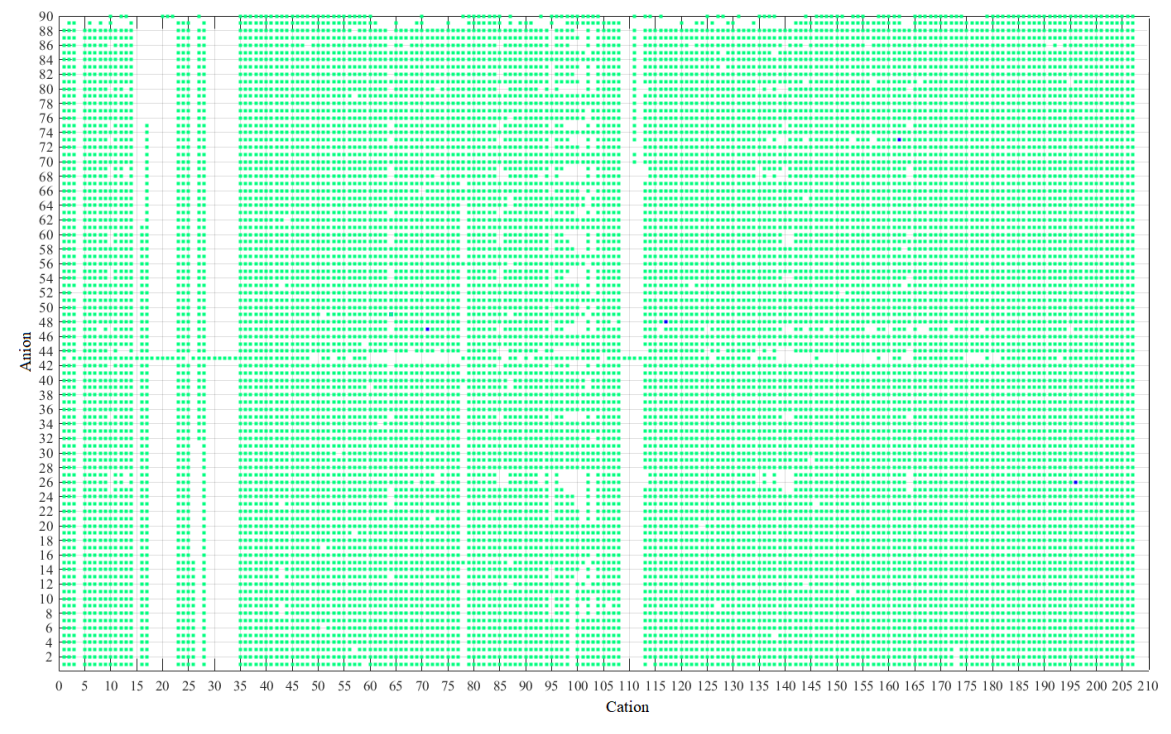

Figure 13. Prediction results of viscosity: green-reasonable, blue-unreasonable.

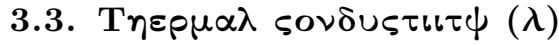

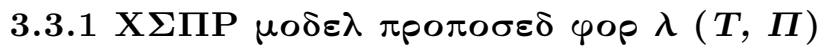

A QSPR model is proposed to predict the thermal conductivity of ILs and expressed as Eq. (22). The parameter values are shown in Supporting Information Table C3.

$n=608, R^{2}=0.9847, F=961, Q^{2}=0.9825, A A R D=1.02 \%, R M S E=0.0026$

Where, $I_{\mathrm{ILs}}, I_{\mathrm{Ca}}$ and $I_{\text {An }}$ represent the norm descriptors of ILs, cation and anion, respectively.

The calculated $\lambda$ data of ILs by Eq. (22) are listed in Supporting Information Table S3 together with the observed $\lambda$ data. Figure 14 (a) presented the comparison between experimental and predicted $\lambda$ of ILs. Distribution of the residual by the model and LOO-CV is shown in Figure 14 (b).
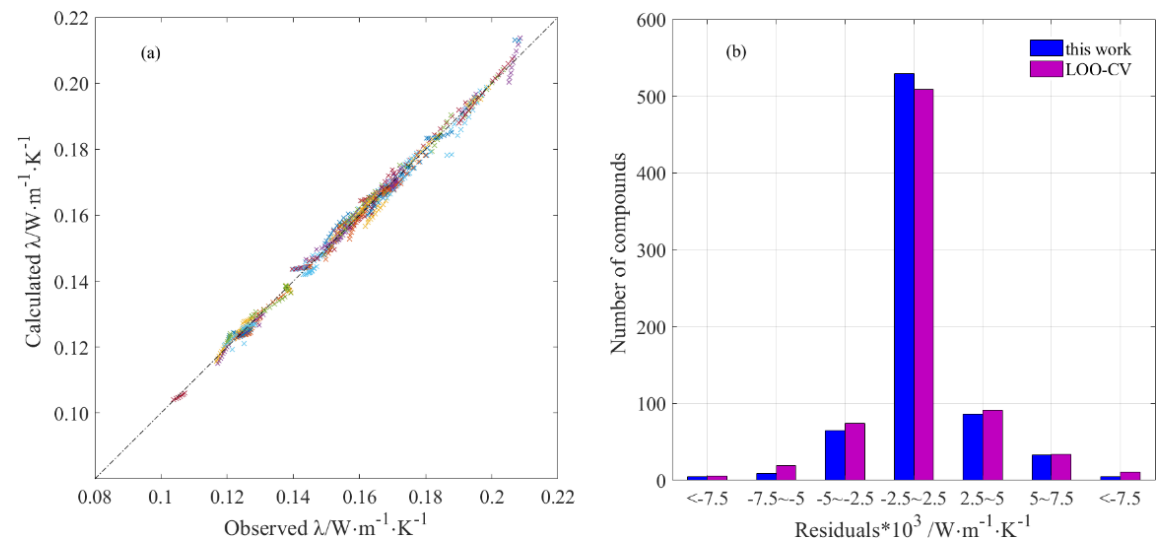

Figure 14. Observed vs. predicted values (a) and distribution of the residual (b) of thermal conductivity 
Figure 14 (a) indicated that the calculated values agreed very well with observed data for $\lambda$ values. Also, the good performance of this model could be demonstrated by the high $R^{2}$ of 0.9847 and the low $A A R D$ of $1.02 \%$. As seen from Figure 14 (b), most of the points fell between -0.0025 and $0.0025 \mathrm{~W} \mathrm{~m}^{-1} \mathrm{~K}^{-1}$, and only a small number of predicted residual errors were greater than $0.0075 \mathrm{~W} \mathrm{~m}^{-1} \mathrm{~K}^{-1}$. Therefore, it might be possible to successfully calculate the $\lambda$ of ILs at variable temperatures and pressures by this model.

Experimental data on the $\lambda$ of 87 ILs were collected in this paper, including 5 classes of cations and 12 classes of anions. It can be easily noted from Figure 15 (a) that, despite the great diversity of the cations and anions of ILs, only a few types of ions have been widely studied. Figure 15 (b) shows that the AARD calculated from all reported data of a given cation / anion group is small and all types of ILs residuals are acceptable range except for $[\operatorname{Im}]\left[\mathrm{N}(\mathrm{CN})_{2}\right]$. Therefore, the model is suitable for accessing the $\lambda$ data of these kinds of ILs with reasonable results.

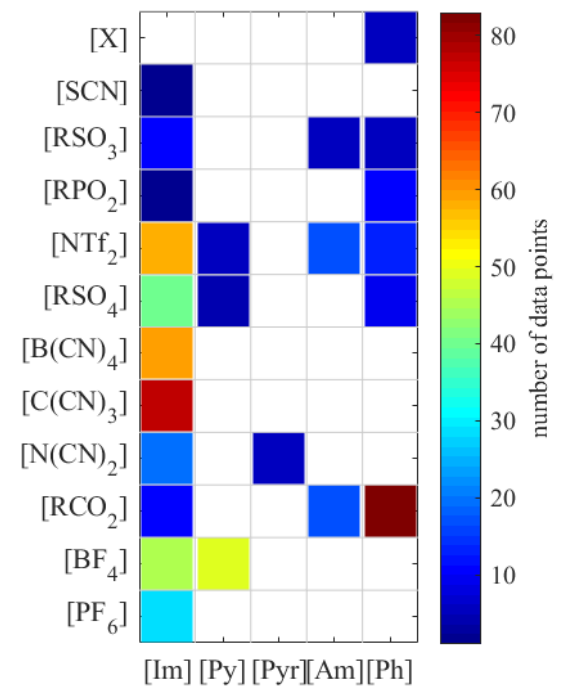

(a)

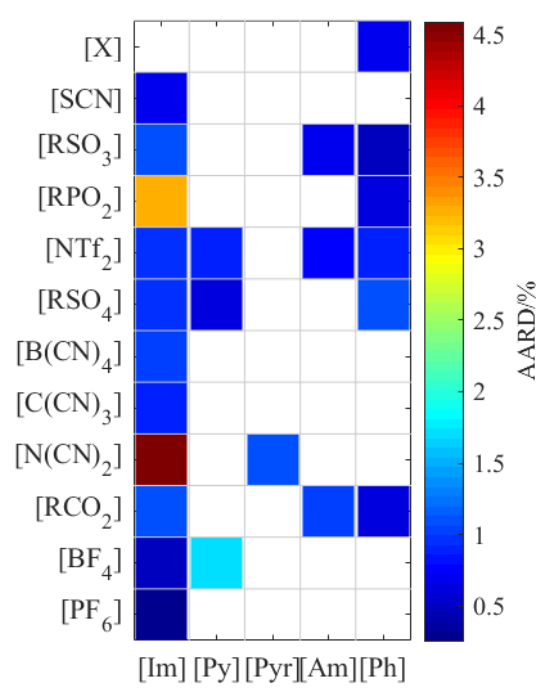

(b)

Figure 15. The numbers (a) and $A A R D \%$ (b) for each kind of ILs for thermal conductivity

\subsubsection{Internal-validation and External-validation}

Figure 16 (a) was plotted with the predicted values by LOO-CV and the experimental $\lambda$ values. The large $Q^{2}(0.9825)$ and the good agreement between the predicted and experimental $\lambda$ further indicated the good stability of this model.

The $\lambda$ data set is divided into the training set (487) and the testing set (121). Results in Figure 16 (b) indicated that the predicted $\lambda$ were in good agreement with the experimental $\lambda$ values of ILs. The statistical parameters listed in Table 5 showed that the $R^{2}$ of training set and testing set were 0.9806 and 0.9834 , and the $A A R D$ were $1.292 \%$ and $1.146 \%$, respectively, which further proved the predictability of this $\lambda$ model. 

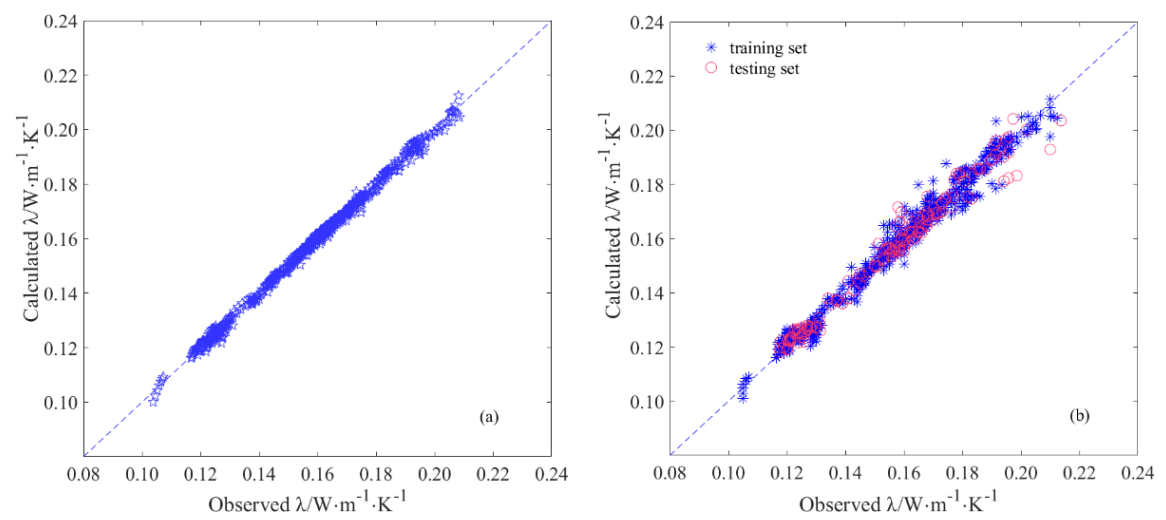

Figure 16. Scatter plots of the internal-validation (a) and external-validation (b)

Table 5. The results of internal-validation and external-validation for thermal conductivity.

\begin{tabular}{lllll}
\hline Status & Data points & $R^{2}\left(Q^{2}\right)$ & $A A R D \%$ & $R M S E$ \\
\hline Model & 608 & 0.9847 & 1.0176 & 0.0026 \\
LOO-CV & 608 & 0.9825 & 1.0192 & 0.0031 \\
Training set & 487 & 0.9806 & 1.2923 & 0.0030 \\
Testing set & 121 & 0.9837 & 1.1457 & 0.0025 \\
\hline
\end{tabular}

\subsubsection{Y -randomization test}

Figure 17 shows the results of 10,000 $Y$-random validations. The $R_{\mathrm{r}}{ }^{2}$ and $Q{ }_{\mathrm{r}}{ }^{2}$ of $Y$-type randomized test were lower than 0.08 , which was significantly smaller than those of the original $\lambda$ model. Therefore, it can be considered that the QSPR model were not affected by the chance correlation. 


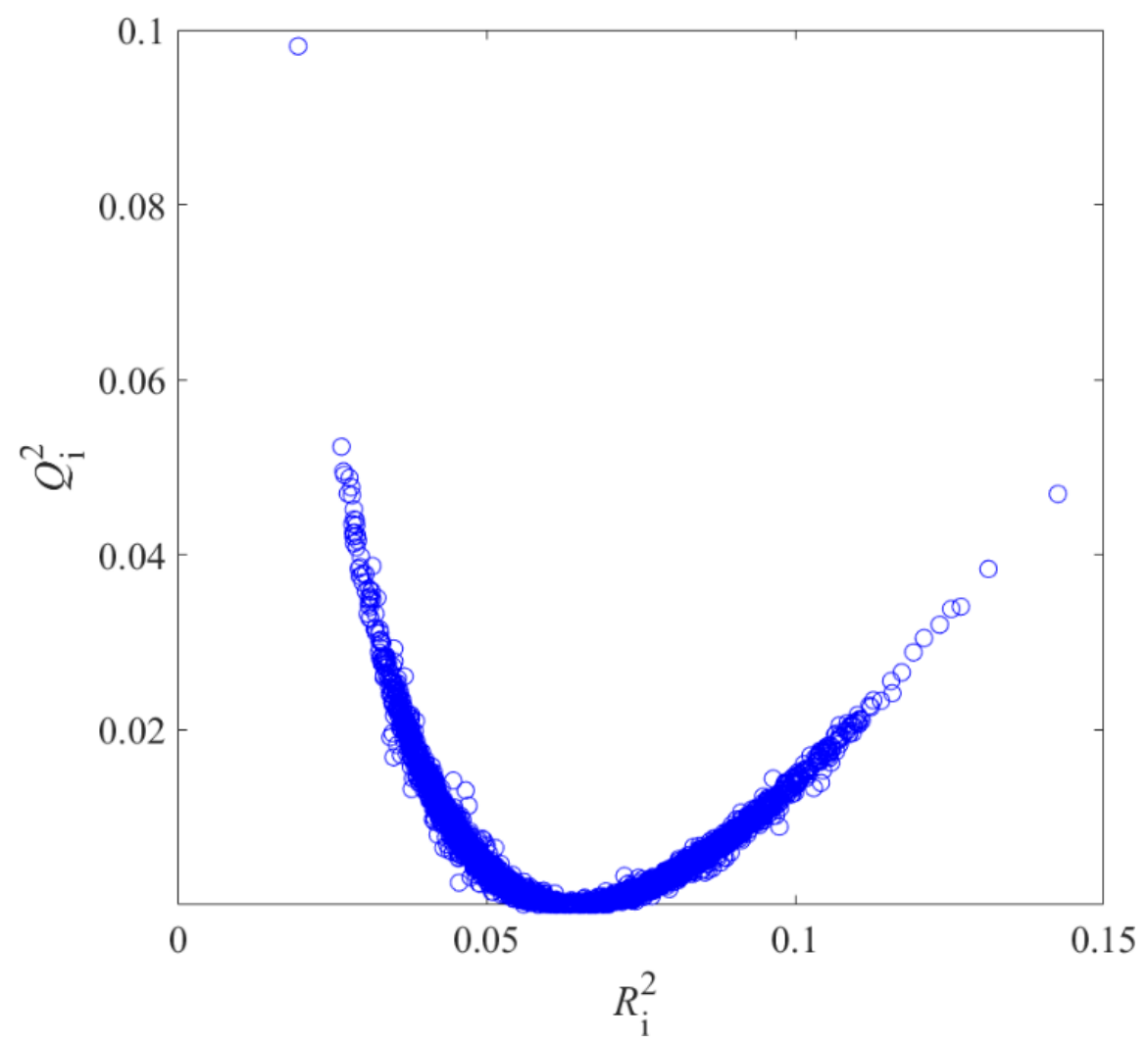

Figure 17. Results of the 10000 times Y-randomization test for thermal conductivity.

\subsubsection{Comparison with References}

Table 6. The comparisons of this work with references for thermal conductivity

\begin{tabular}{|c|c|c|c|c|c|c|c|}
\hline References & Model & D.P & ILs & $\mathrm{T} / \mathrm{K}$ & $\mathrm{P} / \mathrm{MPa}$ & $R^{2}$ & $\begin{array}{l}A A R D \\
(\%)\end{array}$ \\
\hline $\begin{array}{l}\text { Lazzús } \\
2015^{22}\end{array}$ & GCM & 400 & 41 & $273-390$ & $0.1-20$ & 0.9843 & 2.12 \\
\hline $\begin{array}{l}\text { Chen et } \\
\text { al. } 2014^{44}\end{array}$ & QSPR & 359 & 45 & $\begin{array}{l}273.15- \\
353.15\end{array}$ & 1.01 & & 2.30 \\
\hline $\begin{array}{l}\text { Lazzus } \\
2015^{45}\end{array}$ & QSPR & 195 & 20 & 273.15-390 & 1.01 & 0.9919 & 2.00 \\
\hline $\begin{array}{l}\text { He et al. } \\
2017^{56}\end{array}$ & QSPR & 475 & 50 & $\begin{array}{l}273.15- \\
355.07\end{array}$ & $0.1-20$ & 0.9840840 & 1.45 \\
\hline This work & QSPR & 608 & 87 & 273.15-390 & $0.1-20$ & 0.9847 & 1.02 \\
\hline
\end{tabular}

The QSPR model for predicting $\lambda$ is established and compared with other models as shown in Table 6. Lazzús ${ }^{22}$ using GCM to predict the $\lambda$ at variable temperature and pressure and got good results $(R$ ${ }^{2}=0.9843, A A R D=2.12 \%$ ). Compared with Chen et al. ${ }^{44}$, Lazzus ${ }^{45}$ and He et al. ${ }^{56}$, our work has a larger amount of data points, and obtained a higher $R^{2}$ of 0.9847 , a lower $A A R D$ of $1.02 \%$. Therefore, our QSPR model for $\lambda$ prediction has the higher precision.

\subsubsection{The application of QSPR model}


The parameters of thermal conductivity model are located in Supporting Information Table S7. For 16329 ILs, the prediction of ILs thermal conductivity at variable temperature (273-573 K) and pressure (0.1-10 $\mathrm{MPa}$ ) is shown in Figure 18. It can be seen from Figure 18 that this model is suitable for the thermal conductivity calculation of most ILs at variable temperature and atmospheric pressure; the prediction of thermal conductivity of ILs corresponding to these seven anions ([BF4], [ClO4], [Cl], [For], [NO3], [PF6], $[\mathrm{SCN}])$ at other temperatures and pressures is in a reasonable range; however, the thermal conductivity of ILs corresponding to four cations ([(prOH)py], [C11mim], [P1444], [P2228]) and six anions ([BF2Ox], [Br], [C2H5BF3], [C3F7BF3], [C3H7BF3], [I]) is not within the reasonable range.

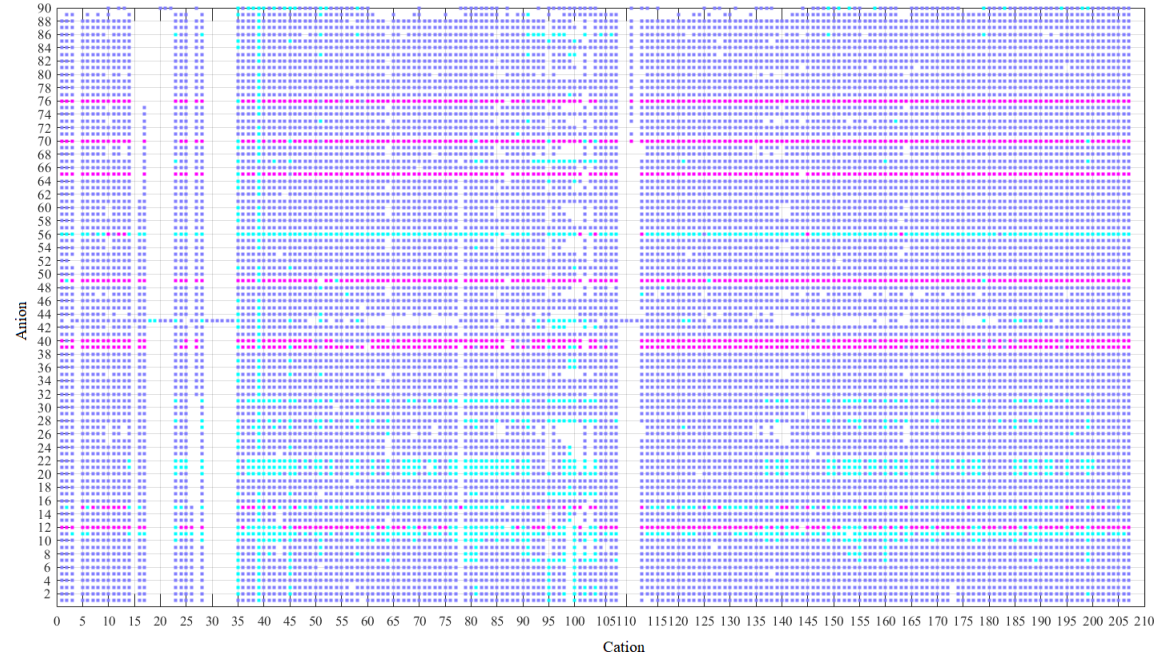

Figure 18. Prediction results of thermal conductivity: purple-reasonable at variable temperature and $0.1 \mathrm{MPa}$;

red-reasonable at variable temperature and pressure, pale blue-unreasonable.

\section{Conclusions}

In this paper, three groups of norm descriptors were established by the structure of ILs, anion and anion. Based on $9020 \rho$ data points, $7324 \eta$ data points and $608 \lambda$ data points, three QSPR models were established to calculate the properties of ILs (density, viscosity and thermal conductivity) at variable temperatures and pressures. The performance of the $f-T-P$ QSPR models was improved by introducing norm descriptors into temperature and pressure terms. The statistical results indicate that these models have good prediction accuracy and credibility with the high $R^{2}$ of $0.9966,0.9676$ and 0.9847 , the low $A A R D$ of $0.48 \%, 3.91 \%$ and $1.02 \%$ for predicting the density, viscosity and thermal conductivity, respectively. Validation analysis shows that these QSPR models have good prediction ability and robustness. Moreover, parameters $(\alpha, \beta, \gamma$ and $\chi$ ) of thef-T-P model for 16329 ILs are predicted by QSPR models and most of the predictions of density and viscosity values of ILs at variable temperature and pressure are within a reasonable range, except for [bmim] [FS], [Hprthiur][I], $\left[\mathrm{mC}_{10} \mathrm{im}\right]\left[\mathrm{Pf}_{2} \mathrm{~N}\right],[\mathrm{N} 113(\mathrm{eOH})][\mathrm{FAP}]$ and $[\mathrm{odbu}]\left[\mathrm{C}_{8} \mathrm{SO}_{3}\right]$. At variable temperature and atmospheric pressure, the predicted thermal conductivities of most ILs are in a reasonable range. At variable temperature and pressure, the predicted thermal conductivity of these ILs corresponding to seven anions are within the reasonable range, and the predictions of these ILs corresponding to four cations and six anions are not in the reasonable range. The prediction results show that the model is stable and widely used.

\section{Supplementary Material}

The fitting parameters $a, \beta, \gamma$ and $\chi$ off-T-P model, used to calculate the density, viscosity and thermal conductivity of ILs are given in Supporting Information Figures S1 ${ }^{\sim}$ S3. The experimental and calculated 
values of density, viscosity and thermal conductivity were listed in Tables S1 $\sim \mathrm{S} 3$. The atomic distribution matrix $(M)$, used for density, viscosity and thermal conductivity, are shown in Supporting Information Tables C1-C3. The coefficients $(\alpha, \beta$, yand $\chi$ ) of density, viscosity and thermal conductivity were predicted and provided in Supporting Information Tables S5-S7. Also, an example for calculating the thermal conductivity with the established model was given.

\section{Conflict interest statement}

The authors confirm that this article has no conflicts of interest.

\section{Acknowledgements}

This work was supported by the National Natural Science Foundation of China [NO: 21808167 and 21676203].

\section{Literature Cited}

1. Cheng Lian, Honglai Liu, Chunzhong Li, and Jianzhong Wu, "Hunting ionic liquids with large electrochemical potential windows," AIChE Journal 65 (2019). 2. Zhongde Dai, Luca Ansaloni, Douglas L. Gin, Richard D. Noble, and Liyuan Deng, "Facile fabrication of CO2 separation membranes by cross-linking of poly(ethylene glycol) diglycidyl ether with a diamine and a polyamine-based ionic liquid," Journal of Membrane Science 523, 551-560 (2017). 3. Christopher P. Fredlake, Jacob M. Crosthwaite, Daniel G. Hert, Sudhir N. V. K. Aki, and Joan F. Brennecke, "Thermophysical Properties of Imidazolium-Based Ionic Liquids," Journal of Chemical \& Engineering Data 49 (4), 954-964 (2004). 4. Zhiqi He and Paschalis Alexandridis, "Ionic liquid and nanoparticle hybrid systems: Emerging applications," Advances in Colloid and Interface Science 244, 54-70 (2017). 5. Tomo Sakanoue, Fumihiro Yonekawa, Ken Albrecht, Kimihisa Yamamoto, and Taishi Takenobu, "An ionic liquid that dissolves semiconducting polymers: a promising electrolyte for bright, efficient, and stable light-emitting electrochemical cells," Chemistry of Materials 29 (14), 6122-6129 (2017). 6. José O. Valderrama and Roberto E. Rojas, "Mass connectivity index, a new molecular parameter for the estimation of ionic liquid properties," Fluid Phase Equilibria297 (1), 107-112 (2010). 7. Yoshihiro Hayashi, Yuki Marumo, Takumi Takahashi, Yuri Nakano, Atsushi Kosugi, Shungo Kumada, Daijiro Hirai, Kozo Takayama, and Yoshinori Onuki, "In silico predictions of tablet density using a quantitative structureproperty relationship model," International Journal of Pharmaceutics 558, 351-356 (2019). 8. Min-Rui Gao, Jiayin Yuan, and Markus Antonietti, "Ionic Liquids and Poly(ionic liquid)s for Morphosynthesis of Inorganic Materials," Chem. - A Europ. J.23 (23), 5391-5403 (2017). 9. Ryosuke Yamada, Kazunori Nakashima, Nanami Asai-Nakashima, Wataru Tokuhara, Nobuhiro Ishida, Satoshi Katahira, Noriho Kamiya, Chiaki Ogino, and Akihiko Kondo, "Direct Ethanol Production from Ionic Liquid-Pretreated Lignocellulosic Biomass by Cellulase-Displaying Yeasts," Appl. Biochem. Biotech. 182(1), 229-237 (2017).

10. Samuel Kassaye, Kamal K. Pant, and Sapna Jain, "Hydrolysis of cellulosic bamboo biomass into reducing sugars via a combined alkaline solution and ionic liquid pretreament steps," Renew. Energ. 104 , 177-184 (2017).

11. Wan-Qi Su, Cheng Yang, and Da-Zhen Xu, "Ionic liquid [Dabco-C8][FeCl4] as an efficient and recyclable catalyst for direct C3 alkylation of indoles with electron-deficient olefins," Catalysis Communications 100 , 38-42 (2017).

12. Isabel Bandrés, Rafael Alcalde, Carlos Lafuente, Mert Atilhan, and Santiago Aparicio, "On the Viscosity of Pyridinium Based Ionic Liquids: An Experimental and Computational Study," The Journal of Physical Chemistry B 115 (43), 12499-12513 (2011).

13. Divya P. Soman, P. Kalaichelvi, and T. K. Radhakrishnan, "THERMAL CONDUCTIVITY ENHANCEMENT OF AQUEOUS IONIC LIQUID AND NANOPARTICLE SUSPENSION," Brazilian Journal of Chemical Engineering 36 , 855-868 (2019).

14. Safar Ali Shojaee, Samira Farzam, Ali Zeinolabedini Hezave, Mostafa Lashkarbolooki, and Shahab Ayatollahi, "A new correlation for estimating thermal conductivity of pure ionic liquids," Fluid Phase Equilibria 354, 199-206 (2013). 
15. Nguyen Minh Quang, Tran Xuan Mau, Nguyen Thi Ai Nhung, Tran Nguyen Minh An, and Pham Van Tat, "Novel QSPR modeling of stability constants of metal-thiosemicarbazone complexes by hybrid multivariate technique: GA-MLR, GA-SVR and GA-ANN," Journal of Molecular Structure 1195 , 95-109 (2019).

16. Y. Q. Chen, G. M. Kontogeorgis, and J. M. Woodley, "Group Contribution Based Estimation Method for Properties of Ionic Liquids," Industrial \& Engineering Chemistry Research 58 (10), 4277-4292 (2019).

17. João A. P. Coutinho, Pedro J. Carvalho, and Nuno M. C. Oliveira, "Predictive methods for the estimation of thermophysical properties of ionic liquids," RSC Advances2 (19), 7322 (2012).

18. Anna Rybinska, Anita Sosnowska, Maciej Barycki, and Tomasz Puzyn, "Geometry optimization method versus predictive ability in QSPR modeling for ionic liquids," Journal of Computer-Aided Molecular Design 30 (2), 165-176 (2016).

19. Wenhui Tu, Lu Bai, Shaojuan Zeng, Hongshuai Gao, Suojiang Zhang, and Xiangping Zhang, "An ionic fragments contribution-COSMO method to predict the surface charge density profiles of ionic liquids," Journal of Molecular Liquids 282, 292-302 (2019).

20. Fabrice Mutelet, Virginia Ortegavilla, Jean Charles Moïse, and Jean Noël Jaubert, "Prediction of Partition Coefficients of Organic Compounds in Ionic Liquids Using a Temperature-Dependent Linear Solvation Energy Relationship with Parameters Calculated through a Group Contribution Method," Unt Scholarly Works 56 (9), 3598-3606 (2011).

21. Kamil Paduszyński and Urszula Domańska, "A New Group Contribution Method For Prediction of Density of Pure Ionic Liquids over a Wide Range of Temperature and Pressure," Ind. Eng. Chem. Res. 51 (1), 591-604 (2012).

22. Juan A. Lazzús, "A group contribution method to predict the thermal conductivity $\lambda(\mathrm{T}, \mathrm{P})$ of ionic liquids," Fluid Phase Equilib. 405 , 141-149 (2015).

23. Alireza Ahmadi, Reza Haghbakhsh, Sona Raeissi, and Vahid Hemmati, "A simple group contribution correlation for the prediction of ionic liquid heat capacities at different temperatures," Fluid Phase Equilibria 403, 95-103 (2015).

24. Wensi He, Fangyou Yan, Qingzhu Jia, Shuqian Xia, and Qiang Wang, "Prediction of ionic liquids heat capacity at variable temperatures based on the norm indexes," Fluid Phase Equilibria 500 , 112260 (2019).

25. Fangyou Yan, Yajuan Shi, Ying Wang, Qingzhu Jia, Qiang Wang, and Shuqian Xia, "QSPR models for the properties of ionic liquids at variable temperatures based on norm descriptors," Chemical Engineering Science 217, 115540 (2020).

26. Guangren Yu, Lu Wen, Dachuan Zhao, Charles Asumana, and Xiaochun Chen, "QSPR study on the viscosity of bis(trifluoromethylsulfonyl)imide-based ionic liquids," J. Mol.Liq.184 , 51-59 (2013).

27. Bor-Kuan Chen, Ming-Jyh Liang, Tzi-Yi Wu, and H. Paul Wang, "A high correlate and simplified QSPR for viscosity of imidazolium-based ionic liquids," Fluid Phase Equilibria350 , 37-42 (2013).

28. Guangren Yu, Dachuan Zhao, Lu Wen, Shendu Yang, and Xiaochun Chen, "Viscosity of ionic liquids: Database, observation, and quantitative structure-property relationship analysis," AIChE Journal $\mathbf{5 8}$ (9), 2885-2899 (2012).

29. Fangyou Yan, Wensi He, Qingzhu Jia, Qiang Wang, Shuqian Xia, and Peisheng Ma, "Prediction of ionic liquids viscosity at variable temperatures and pressures," Chemical Engineering Science 184, 134-140 (2018).

30. Johannes Albert and Karsten Müller, "A Group Contribution Method for the Thermal Properties of Ionic Liquids," Industrial \& Engineering Chemistry Research 53 (44), 17522-17526 (2014). 
31. Wanqiang Liu, Haixia Lu, Chenzhong Cao, Yinchun Jiao, and Guanfan Chen, "An Improved Quantitative Structure Property Relationship Model for Predicting Thermal Conductivity of Liquid Aliphatic Alcohols," J. Chem. Eng. Data.63 (12), 4735-4740 (2018).

32. Karim Golzar, Sepideh Amjad-Iranagh, and Hamid Modarress, "Prediction of Density, Surface Tension, and Viscosity of Quaternary Ammonium-Based Ionic Liquids ([N222(n)]Tf2N) by Means of Artificial Intelligence Techniques," Journal of Dispersion Science and Technology 35 (12), 1809-1829 (2014).

33. Łukasz Marcinkowski, Emil Szepiński, Maria J. Milewska, and Adam Kloskowski, "Density, sound velocity, viscosity, and refractive index of new morpholinium ionic liquids with amino acid-based anions: Effect of temperature, alkyl chain length, and anion," J. Mol.Liq. 284, 557-568 (2019).

34. V. E. Kuz'min, E. N. Muratov, A. G. Artemenko, L. Gorb, M. Qasim, and J. Leszczynski, "The effect of nitroaromatics' composition on their toxicity in vivo: Novel, efficient non-additive 1D QSAR analysis," Chemosphere 72 (9), 1373-1380 (2008).

35. Kunal Roy, Rudra Narayan Das, and Paul L. A. Popelier, "Quantitative structure-activity relationship for toxicity of ionic liquids to Daphnia magna: Aromaticity vs. lipophilicity," Chemosphere 112, 120-127 (2014).

36. Kunal Roy, Rudra Narayan Das, and Paul L. A. Popelier, "Predictive QSAR modelling of algal toxicity of ionic liquids and its interspecies correlation with Daphnia toxicity," Environmental Science and Pollution Research 22 (9), 6634-6641 (2015).

37. Wensi He, Fangyou Yan, Qingzhu Jia, Shuqian Xia, and Qiang Wang, "QSAR models for describing the toxicological effects of ILs against Staphylococcus aureus based on norm indexes," Chemosphere 195 , 831-838 (2018).

38. J. Lazzús, " $\rho(T, p)$ model for ionic liquids based on quantitative structure-property relationship calculations," J. Phys. Org. Chem. 22, 1193 (2009).

39. Fangyou Yan, Qiaoyan Shang, Shuqian Xia, Qiang Wang, and Peisheng Ma, "Application of Topological Index in Predicting Ionic Liquids Densities by the Quantitative Structure Property Relationship Method," J. Chem. Eng. Data. 60(3), 734-739 (2015).

40. Kamil Claudia L. AguirrePaduszynski, "Extensive Databases and Group Contribution QSPRs of Ionic Liquids Properties. 1. Density," Industrial \& engineering chemistry process design and development 2019 v.58 no.13 (no. 13), pp. 5322-5338 (2019).

41. Kamil Paduszyński, "Extensive Databases and Group Contribution QSPRs of Ionic Liquids Properties. 2. Viscosity," Ind. Eng. Chem. Res. 58 (36), 17049-17066 (2019).

42. Wesley Beckner, Coco M. Mao, and Jim Pfaendtner, "Statistical models are able to predict ionic liquid viscosity across a wide range of chemical functionalities and experimental conditions," Molecular Systems Design \& Engineering3 (1), 253-263 (2018).

43. Yongsheng Zhao, Ying Huang, Xiangping Zhang, and Suojiang Zhang, "A quantitative prediction of the viscosity of ionic liquids using S[sigma]-profile molecular descriptors," Phys. Chem. Chem. Phys. 17 (5), 3761-3767 (2015).

44. Qiao-Li Chen, Ke-Jun Wu, and Chao-Hong He, "Thermal Conductivity of Ionic Liquids at Atmospheric Pressure: Database, Analysis, and Prediction Using a Topological Index Method," Ind. Eng. Chem. Res. 53 (17), 7224-7232 (2014).

45. Juan A. Lazzús and Geraldo Pulgar-Villarroel, "Estimation of thermal conductivity of ionic liquids using quantitative structure-property relationship calculations," J. Mol. Liq. 211 , 981-985 (2015).

46. Wensi He, Fangyou Yan, Qingzhu Jia, Shuqian Xia, and Qiang Wang, "Description of the Thermal Conductivity $\lambda(T, P)$ of Ionic Liquids Using the Structure-Property Relationship Method," Journal of Chemical 
\& Engineering Data62 (8), 2466-2472 (2017).

47. Fangyou Yan, Shuqian Xia, Qiang Wang, Zhen Yang, and Peisheng Ma, "Predicting the melting points of ionic liquids by the Quantitative Structure Property Relationship method using a topological index," The Journal of Chemical Thermodynamics62，196-200 (2013).

48. Fangyou Yan, Tian Lan, Xue Yan, Qingzhu Jia, and Qiang Wang, "Norm index-based QSTR model to predict the eco-toxicity of ionic liquids towards Leukemia rat cell line," Chemosphere 234 , 116-122 (2019).

49. Christoph Rücker, Gerta Rücker, and Markus Meringer, "y-Randomization and Its Variants in QSPR/QSAR," Journal of Chemical Information and Modeling 47 (6), 2345-2357 (2007).

50. Kunal Roy, Indrani Mitra, Supratik Kar, Probir Kumar Ojha, Rudra Narayan Das, and Humayun Kabir, "Comparative Studies on Some Metrics for External Validation of QSPR Models," Journal of Chemical Information and Modeling 52 (2), 396-408 (2012).

51. Ali Barati-Harooni, Adel Najafi-Marghmaleki, Milad Arabloo, and Amir H. Mohammadi, "An accurate CSA-LSSVM model for estimation of densities of ionic liquids," Journal of Molecular Liquids 224 , 954-964 (2016).

52. Juan A. Lazzús, "A GROUP CONTRIBUTION METHOD TO PREDICT p-T-P OF IONIC LIQUIDS," Chemical Engineering Communications 197 (7), 974-1015 (2010).

53. Juan A. Lazzús, " $-\mathrm{T}-\mathrm{P}$ prediction for ionic liquids using neural networks," Journal of the Taiwan Institute of Chemical Engineers 40 (2), 213-232 (2009).

54. Fangyou Yan, Qiaoyan Shang, Shuqian Xia, Qiang Wang, and Peisheng Ma, "Application of Topological Index in Predicting Ionic Liquids Densities by the Quantitative Structure Property Relationship Method," Journal of Chemical \& Engineering Data 60 (3), 734-739 (2015).

55. Yongsheng Zhao, Ying Huang, Xiangping Zhang, and Suojiang Zhang, "A quantitative prediction of the viscosity of ionic liquids using S $\sigma$-profile molecular descriptors," Physical Chemistry Chemical Physics $\mathbf{1 7}$ (5), 3761-3767 (2015).

56. Wensi He, Fangyou Yan, Qingzhu Jia, Shuqian Xia, and Qiang Wang, "Description of the thermal conductivity $\lambda(\mathrm{T}, \mathrm{P})$ of ionic liquids using the structure-property relationship method," Journal of Chemical \& Engineering Data62 (8), 2466-2472 (2017). 